\title{
A comunicação entre o casal no contexto do cancro da mama
}

\author{
Communication between the couple \\ in the context of breast cancer
}

\author{
Helena MOREIRA ${ }^{1}$ \\ Maria Cristina CANAVARRO
}

\begin{abstract}
Resumo
A comunicação entre o casal - a partilha de pensamentos, sentimentos e preocupações -, é fundamental para o bem-estar, individual e relacional, de cada elemento da díade conjugal. No contexto de uma doença como o cancro da mama, essa partilha adquire particular relevância, nomeadamente pelo seu importante papel no processamento cognitivo da experiência de cancro e, portanto, na adaptação bem-sucedida à doença. Contudo, a investigação não se tem centrado especificamente no estudo da comunicação em doentes com cancro da mama, nem tão pouco na sistematização e integração da informação existente. O presente estudo tem como objetivo rever criticamente a literatura sobre o tema, procurando integrar diversos resultados num todo que seja coerente e útil para quem investiga esta área ou trabalha com casais na situação descrita.
\end{abstract}

Palavras-chave: Adaptação; Casal; Comunicação; Neuplasias da mama.

\begin{abstract}
Communication between the couple, i.e., the sharing of thoughts, feelings and concerns, is crucial for the individual and relational well-being of each element of the marital dyad. In the context of a disease such as breast cancer, this sharing is particularly relevant, especially because of its important role in the cognitive processing of the cancer experience and, therefore, the successful adaptation to the disease. However, research has not specifically focused on the study of communication in patients with breast cancer, or on the systematization and integration of existing information. The present study aimed to critically review the existing literature on this topic, and to integrate the various results into a whole that is coherent and useful for those who study this area and/or work with these couples.
\end{abstract}

Keywords: Adaptation; Couple; Communication; Breast neoplasms.

Ainda que a comunicação entre o casal no contexto da doença oncológica e, especificamente, do cancro da mama, seja apontada por alguns investigadores (Manne et al., 2006; Porter, Keefe,

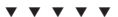

1 Universidade de Coimbra, Faculdade de Psicologia e de Ciências da Educação, Instituto de Psicologia Cognitiva e Desenvolvimento Vocacional e Social. R. do Colégio Novo, Apartado 6153, 3001-802, Coimbra, Portugal. Correspondência para/Correspondence to: H. MOREIRA. E-mail: <hmoreira@fpce.uc.pt>

Artigo elaborado a partir da tese de H. MOREIRA, intitulada "Imagem Corporal, relação de casal e adaptação de mulheres com cancro da mama". Universidade de Coimbra, Portugal, 2010.
} 
Hurwitz, \& Faber, 2005) como um importante aspecto na adaptação do casal à doença, sua investigação tem recebido pouca atenção na literatura. O mesmo se verifica relativamente ao seu estudo em contextos mais abrangentes, como a família ou outras relações sociais, apesar de alguns estudos evidenciarem o importante papel da partilha de experiências e preocupações também nesses contextos.

Sendo o companheiro apontado pelas doentes como o confidente mais importante da sua rede social (Figueiredo, Fries, \& Ingram, 2004), o presente estudo tem como principal objetivo rever a literatura referente à comunicação entre o casal, de modo a integrar e sistematizar alguns dos principais resultados obtidos nos estudos desenvolvidos nessa área. Assim, a presente revisão encontra-se organizada em três pontos distintos. O primeiro procura evidenciar a importância da comunicação na adaptação da doente com cancro, na adaptação do companheiro e no funcionamento da própria relação, e apresenta algumas explicações para o papel positivo que desempenha. A seguir, é dada especial atenção aos modelos de processamento cognitivo dos acontecimentos traumáticos, e procura-se demonstrar o papel central da comunicação no processamento da experiência de cancro e, consequentemente, na adaptação. São também abordados alguns obstáculos à comunicação e, desse modo, ao processamento da informação, nomeadamente os constrangimentos sociais e a estratégia de protective buffering.

\section{O papel da comunicação na adaptação ao cancro da mama}

Alguns estudos têm demonstrado que a qualidade e a quantidade de informação sobre a doença que é partilhada entre o casal ou mesmo no contexto familiar ou no de outras redes sociais mais abrangentes têm um impacto significativo na adaptação psicossocial da doente e do seu companheiro (Cordova, Cunningham, Carlson, \& Andrykowski, 2001; Pistrang \& Barker, 1992; 1995), bem como na qualidade da sua relação conjugal
Cordova et al. (2001) verificaram que as doentes com cancro da mama que partilhavam com frequência seus sentimentos relacionados com a doença reportavam níveis inferiores de depressão e menor distress psicológico. Também Spiegel, Bloom e Gottheil (1983) verificaram, numa amostra de doentes com cancro da mama, que uma atmosfera familiar na qual era encorajada a discussão aberta de sentimentos e problemas predizia menor perturbação do humor durante o ano subsequente. De igual modo, Mesters et al. (1997) observaram, numa amostra de doentes com cancro da mama, da cabeça e do pescoço e de Hodgkin, que os doentes pertencentes a famílias nas quais podiam falar abertamente sobre a doença apresentavam uma adaptação psicossocial mais positiva, ou seja, reportavam menos medo e ansiedade, sentiam-se menos inseguros e deprimidos, referiam um menor sentimento de solidão, sentiam um maior controle e autoestima e manifestavam menos queixas psicológicas e físicas.

A adaptação da mulher com cancro da mama está também relacionada com a forma como o casal resolve problemas relacionados com a doença ou como discute e negocia eventuais fatores de estresse, ou seja, com as suas estratégias de comunicação. Essas estratégias e sua relação com a adaptação à doença têm sido escassamente estudadas em doentes oncológicos e, em particular, em doentes com cancro da mama, não obstante a sua relevância. Procurando colmatar essa lacuna na investigação, Manne et al. (2006) desenvolveram um estudo longitudinal com mulheres com cancro da mama, no qual procuraram analisar a relação entre a adaptação psicológica e a satisfação conjugal e três tipos de estratégias de comunicação: (1) comunicação mutuamente construtiva (discussão mútua das questões, expressão de sentimentos, compreensão do ponto de vista do outro); (2) evitamento mútuo (evitamento mútuo da discussão e afastamento mútuo); e (3) exigência-afastamento (pressão para que o companheiro discuta um problema seguida do seu afastamento). Os autores verificaram que a primeira estratégia estava associada a níveis inferiores de perturbação psicológica e a maior satisfação conjugal nos dois elementos da 
díade; contrariamente, a estratégia de exigência-afastamento associava-se a maior perturbação psicológica e menor satisfação conjugal, igualmente em ambos os elementos; por fim, o evitamento mútuo, embora estivesse associado a níveis superiores de perturbação, não se relacionava com a qualidade da relação conjugal.

Assim, como evidenciado pelo estudo de Manne et al. (2006), a forma como os casais se comunicam, particularmente sobre aspectos associados com a doença, desempenha um papel muito importante na adaptação individual e relacional da doente e do seu companheiro. Importa, por isso, compreender por que é a comunicação tão importante na adaptação de ambos ao cancro da mama. Embora a literatura ainda não tenha oferecido respostas definitivas e consensuais, alguns aspectos merecem ser destacados.

Na revisão da literatura efetuada, encontraram-se algumas razões subjacentes ao papel positivo da comunicação, que irão ser apresentadas. Por exemplo, já na década de 1980, Silver e Wortman (1980) sugeriram que a revelação e a expressão de sentimentos facilitam a adaptação, o bem-estar e as estratégias de coping dos doentes por meio de três vias distintas. Em primeiro lugar, diminuem a tensão emocional e a ansiedade. Em segundo, permitem ao doente receber feedback dos outros acerca das suas reações, normalizando a sua experiência. Por fim, permitem também uma melhor resolução dos problemas, na medida em que o doente pode passar a ver a sua situação a partir de uma perspetiva diferente, mais ampla e objetiva.

Paralelamente, a comunicação parece ser benéfica também para o casal na medida em que aumenta a probabilidade de a doente e o seu companheiro se sentirem mais íntimos e próximos emocionalmente (Porter et al., 2005). Efetivamente, os principais modelos teóricos da intimidade, nomeadamente o modelo de processo interpessoal da intimidade de Reis e Patrick (1996), consideram a comunicação um elemento central da construção e manutenção da intimidade conjugal (Prager, 1995; Manne \& Badr, 2008; Reis \& Patrick, 1996), que, por sua vez, está associada a uma maior satisfação conjugal (Merves-Okin, Edmund, \& Frank, 1991).
Em doentes com cancro, a partilha de sentimentos e pensamentos parece também estar associada a níveis superiores de intimidade, empatia e satisfação conjugal, fatores que conduzem, subsequentemente, a uma melhor adaptação relacional e individual (Giese-Davis, Hermanson, Koopman, Weibel, \& Spiegel, 2000; Pistrang \& Barker, 1995). No estudo de Porter et al. (2005), com doentes com cancro gastrointestinal e seus companheiros, observou-se que quando os doentes reportavam níveis superiores de comunicação, tanto o doente como o seu parceiro apresentavam maior intimidade na relação. Contrariamente, quando os doentes não partilhavam tanto sua experiência com o parceiro, a relação era classificada por ambos como menos íntima, e os companheiros eram descritos pelo doente como menos empáticos, mais críticos e evitantes.

Por fim, é importante destacar que a importância da comunicação para a adaptação do doente pode relacionar-se, em parte, com o fato de esse comportamento facilitar o processamento da informação relacionada com a experiência de cancro, possibilitando ao doente atribuir um novo significado à doença e, desse modo, adaptar-se à situação (Lepore \& Helgeson, 1998; Lepore, Ragan, \& Jones, 2000). Pela sua relevância, esse aspecto será abordado em maior detalhe no ponto seguinte do presente trabalho.

\section{A importância da comunicação e os modelos de processamento cognitivo dos acontecimentos traumáticos}

Os modelos de processamento cognitivo de um acontecimento traumático oferecem um enquadramento teórico adequado para a compreensão da relevância da partilha de informações relacionadas com o cancro, perspectivado, neste contexto, como um acontecimento traumático. A seguir, serão apresentados sucintamente os principais pontos-chave desses modelos, procurando-se enfatizar o papel da comunicação e evidenciar os seus potenciais benefícios. Uma revisão detalhada da literatura concernente às teorias de processamento cognitivo das reações pós-traumáticas encontra-se para além do âmbito do presente trabalho. 
De acordo com os modelos de processamento cognitivo do acontecimento traumático (Creamer, Burgess, \& Pattison, 1992; Foa, Steketee, \& Rothbaum, 1989; Horowitz, 1986; Janoff-Bulman, 1992), os indivíduos apresentam esquemas mentais que contêm informação acerca de si próprios, dos outros e do mundo. São frequentes, por exemplo, as crenças de que se é invulnerável ou de que o mundo é um lugar justo (Lepore, Silver, Wortman, \& Wayment, 1996). Uma experiência traumática, tal como uma doença ameaçadora de vida, desafia as assunções e as crenças nucleares do indivíduo (Janoff-Bulman, 1992). Por exemplo, o cancro da mama, pela natureza imprevisível e ameaçadora de vida, bem como pelas mudanças que impõe no dia a dia da doente, faz com que a pessoa questione frequentemente as crenças nucleares que mantém sobre si própria, sobre o mundo e sobre o futuro (Lepore \& Helgeson, 1998).

Deste modo, à semelhança de outras experiências traumáticas em que o indivíduo vai procurar integrar a informação relacionada com o trauma nos esquemas mentais pré-existentes (Horowitz, 1986), também o doente com cancro necessita integrar a experiência da doença nos seus modelos mentais prévios (Lepore \& Helgeson, 1998). Esse processo, que conduz à adaptação (Lepore \& Helgeson, 1998; Lepore et al., 2000), é designado por processamento cognitivo do acontecimento, e, de acordo com Lepore e Helgeson (1998), refere-se às atividades mentais que ajudam o indivíduo a interpretar os acontecimentos traumáticos e a integrar aspectos ameaçadores ou confusos da experiência num quadro conceptual coerente e não ameaçador. Ou seja, de acordo com os modelos de processamento cognitivo, a recuperação de um acontecimento traumático requer que o indivíduo processe a informação relacionada com o trauma até ser incorporada nos esquemas pré-existentes ou até estes serem modificados de forma a acomodarem a nova informação.

Dois processos parecem estar envolvidos nessa tentativa de integração das experiências traumáticas (Lepore et al., 1996). O primeiro diz respeito à exposição repetida a pensamentos, me100 mórias e imagens relacionadas com o trauma
(Rachman, 1980). Nesta perspectiva, os pensamentos intrusivos podem ser vistos como uma manifestação e atividade central do processamento cognitivo (Horowitz, 1986; Rachman, 1980). Creamer et al. (1992) consideram que a exposição repetida ao estímulo traumático pode levar à modificação do significado associado ao acontecimento. Ou seja, a confrontação, a contemplação e a reavaliação da experiência de cancro podem ajudar o doente a reestruturar as suas crenças nucleares e expectativas, promovendo a integração da experiência na sua visão do self e do mundo (Lepore \& Helgeson, 1998). Assim, segundo Creamer et al. (1992), ainda que os pensamentos intrusivos possam ser perturbadores, a longo prazo são adaptativos, pois a exposição a essas memórias relacionadas com o trauma permite o enfraquecimento das ligações estímulo-resposta e conduz à modificação do significado associado ao trauma.

O segundo processo, que pode ser considerado uma estratégia facilitadora do primeiro, passa pela comunicação ou revelação aos outros do acontecimento traumático ou de aspectos relacionados com este. De fato, esse processo de comunicação ou partilha da experiência traumática foi, desde sempre, encarado como um mecanismo facilitador da adaptação e da recuperação emocional. Pennebaker (1989) evidenciou que a não comunicação sobre experiências traumáticas, como a morte de um ente querido, abuso sexual ou divórcio, estava associada a uma maior perturbação psicológica, procura de ajuda médica e ruminações relacionadas com o trauma. Também no contexto de uma doença oncológica, a comunicação assume, evidentemente, uma grande importância, e facilita a adaptação à doença.

A partir da revisão efetuada, constatou-se que este processo de comunicação pode facilitar o processamento cognitivo e a adaptação ao trauma de diversas formas (Lepore \& Helgeson, 1998; Lepore et al., 1996). Em primeiro lugar, constitui-se uma oportunidade de validação de pensamentos e sentimentos e mostra ao indivíduo que o outro se preocupa com ele. Em segundo lugar, encoraja o indivíduo a desenvolver novas perspectivas sobre a situação (Clark, 1993), ajudando-o a encontrar um 
significado para a experiência e oferecendo-lhe informações sobre como controlar as suas emoções e lidar com os seus problemas. Por fim, pode reduzir os níveis de ativação e, consequentemente, facilitar a integração cognitiva do trauma (Rachman, 1980).

Importa ainda referir que, segundo Lepore et al. (2000), a expressão de pensamentos e sentimentos pode ter impacto na adaptação através do seu efeito nos pensamentos intrusivos. Assim, os autores consideram que os comportamentos de expressão, como falar ou escrever sobre o acontecimento traumático, podem ajudar o indivíduo a construir uma narrativa coerente sobre o sucedido, o que lhe permitirá torná-lo compreensível para si e para os outros. Essa narrativa torna-se parte da representação cognitiva da experiência, alargando, desse modo, a perspectiva do indivíduo e mudando o conteúdo dos pensamentos e das memórias relacionados com o trauma. Se a expressão facilita a reestruturação cognitiva, deve então diminuir a frequência e a intensidade dos pensamentos intrusivos e facilitar a adaptação.

\section{Obstáculos à comunicação}

Apesar do papel positivo da partilha de sentimentos, preocupações, medos e pensamentos relacionados à doença na adaptação individual e relacional da doente, surgem, por vezes, obstáculos a essa partilha tanto no contexto da relação de casal como nos outros contextos relacionais (Manne et al., 2007).

\section{Constrangimentos sociais}

Ainda que a maior parte dos indivíduos deseje falar sobre o acontecimento traumático, muitos experienciam aquilo a que vários autores designam por constrangimentos sociais, que o inibem de falar ou mesmo pensar acerca da sua experiência (Cordova et al., 2001; Lepore \& Helgeson, 1998; Lepore et al., 1996; Lepore et al., 2000). Alguns exemplos são: (1) a falta de acesso a pessoas dispostas a ouvir o indivíduo de forma empática; (2) a manifestação de comportamentos percepcionados como inapropriados ou insensíveis por parte da rede social do indivíduo (e.g., quando a outra pessoa não sabe o que dizer ou fazer e responde de forma inadequada ou minimizadora do acontecimento); (3) a resposta negativa dos outros à partilha do indivíduo (e.g., perante alguns acontecimentos, algumas pessoas podem abandonar ou evitar o indivíduo por se sentirem inúteis ou por não saberem lidar com a situação).

Simultaneamente, pode-se pensar que os constrangimentos sociais têm um impacto negativo no processamento e na integração da experiência. De fato, alguns estudos têm mostrado que, no contexto de uma doença oncológica, a doença pode ter um efeito negativo na qualidade das relações interpessoais, o que, por sua vez, pode impossibilitar o doente de receber a validação e o apoio de que necessita (Dunkel-Schetter \& Wortman, 1982). De acordo com os modelos de processamento cognitivo dos acontecimentos traumáticos, as reações sociais negativas e não apoiantes (crítica, afastamento) podem levar o indivíduo a inibir ou a suprimir os pensamentos relacionados com o trauma, interferindo, assim, no seu processamento cognitivo. Cordova et al. (2001), por exemplo, verificaram, numa amostra de doentes com cancro da mama, que os constrangimentos sociais estavam associados a pontuações superiores nos indicadores autorrelatados de inibição do processamento. Por sua vez, a inibição do processamento, refletida por uma maior incerteza, intrusões, evitamento e menor comunicação, estava relacionada com um nível mais elevado de depressão e menor bem-estar.

Do mesmo modo, em estudo conduzido com indivíduos com cancro da próstata, Lepore e Helgeson (1998) verificaram que os doentes que sentiam mais constrangimentos sociais na comunicação sobre a doença apresentavam maior número de pensamentos intrusivos e evitavam falar e pensar sobre a sua experiência. Constatou-se ainda que os pensamentos intrusivos estavam negativamente associados à saúde mental, particularmente nos indivíduos que reportavam maior número de constrangimentos. Por fim, os autores observaram também que os constrangimentos sociais por parte da companheira eram um preditor mais forte da saúde mental do que os constrangimentos por parte de amigos e família. 
Os dois estudos mencionados parecem apoiar, assim, os modelos de processamento cognitivo dos acontecimentos traumáticos, realçando o importante papel da comunicação e, consequentemente, dos constrangimentos sociais no processamento cognitivo e na adaptação da doente.

\section{A estratégia de protective buffering}

Uma das razões que pode estar subjacente às dificuldades de comunicação no casal é o desejo, por parte de um dos elementos da díade, de não perturbar o parceiro ou incomodá-lo com os seus receios e preocupações. Nesse sentido, Coyne e Smith (1991) descreveram uma estratégia de coping focada na relação, que designaram por protective buffering, e que definiram como "a matter of hiding concerns, denying worries, and yielding to the partner to avoid disagreements" (p.405).

Apesar de a intenção subjacente ao uso dessa estratégia ser a promoção do bem-estar do companheiro (doente ou não) e a sua proteção quanto às preocupações relacionadas com a doença, com o objetivo último de diminuir ou evitar a sua perturbação emocional, alguns estudos têm mostrado que essa estratégia não traz benefícios ao doente, ao companheiro ou ao casal (Coyne \& Smith, 1991; Hagedoorn et al., 2000; Kuijer et al., 2000; Manne et al., 2007). No estudo de Coyne e Smith (1991), com doentes que tinham sofrido de enfarte do miocárdio, observou-se que um maior envolvimento nessa estratégia, tanto por parte do companheiro como do doente, estava associado a níveis mais elevados de perturbação emocional.

Entre doentes com cancro e seus companheiros, Manne, Dougherty, Veach e Kless (1999) observaram que quando as esposas e doentes do sexo feminino utilizavam essa estratégia, três meses depois apresentavam maior perturbação psicológica. Num outro estudo (Manne et al., 2007), apenas com doentes com cancro da mama e seus companheiros, os mesmos autores verificaram que o uso dessa estratégia por parte da doente predizia maior perturbação emocional, tanto nas doentes 102 como nos companheiros.
O protective buffering foi também estudado no contexto de outras estratégias de apoio por parte do companheiro. De acordo com Coyne, Ellard e Smith (1990), existem três formas ou estilos de apoio através dos quais os companheiros saudáveis podem proporcionar apoio ao doente: para além do protective buffering, os autores mencionam o envolvimento ativo (active engagement) e a sobreproteção (overprotection). A estratégia de envolvimento ativo passa pelo envolvimento do outro nas discussões, por lhe perguntar como se sente, se precisa de ajuda ou informação e pelo uso de métodos construtivos de resolução de problemas. Essa estratégia tem sido associada a uma maior satisfação conjugal e à melhoria da qualidade da relação percebida ao longo do curso da doença (Kuijer et al., 2000). Já a sobreproteção relaciona-se com a subestimação das capacidades do doente, resultando, usualmente, numa ajuda excessiva e desnecessária e/ou em tentativas de restrição das atividades do doente. Essa estratégia é frequentemente utilizada quando os companheiros não se sentem autoconfiantes no apoio dado (Hagedoorn et al., 2000) ou quando sentem que o doente tem dificuldades em lidar com a doença (Coyne et al., 1990; Kuijer et al., 2000). Alguns estudos têm mostrado que essa estratégia diminui a autoeficácia do doente (Coyne et al., 1990), bem como o sentimento de controle sobre a sua vida e sobre os resultados da doença (Kuijer et al., 2000). Ainda que Coyne e Smith (1991) tenham estudado essas três formas de apoio essencialmente em casais após enfarte de miocárdio, é plausível pensar que os mesmos processos possam ocorrer em casais que se confrontam com outras doenças, tal como o cancro. Hagedoorn et al. (2000) investigaram o papel dessas três estratégias na satisfação conjugal de doentes com vários tipos de cancro e seus companheiros, e observaram que, enquanto o envolvimento ativo se associava positivamente com a satisfação conjugal, o protective buffering e a sobreproteção correlacionavam-se negativamente com essa variável.

Também Kuijer et al. (2000) conduziram um estudo com doentes com cancro da mama, no qual procuraram analisar a influência dos três tipos de estratégias mencionadas na adaptação das doentes. 
De forma geral, verificaram que tanto a estratégia de envolvimento ativo como a de protective buffering não se associavam significativamente aos níveis de perturbação emocional do doente, ao contrário da sobreproteção, que se associava negativa e significativamente ao bem-estar da mulher e à percepção de controle sobre a doença.

Não obstante o fato de o protective buffering ser utilizado com o objetivo de reduzir ou impedir a perturbação emocional do outro no contexto de uma relação próxima, tal como os estudos mencionados indicam, os resultados são, habitualmente, negativos. Os modelos de processamento do trauma, apresentados anteriormente, permitem compreender a ineficácia desse processo. Com efeito, a não partilha de preocupações ou de informações relacionadas com a doença e com a supressão de sentimentos e pensamentos negativos impede ou, pelo menos, dificulta o processamento cognitivo e emocional do acontecimento, interferindo negativamente na adaptação do sujeito a esse mesmo acontecimento (Lepore et al., 1996).

Paralelamente, para além do impacto negativo dessa estratégia para a pessoa que a utiliza, segundo Manne et al. (2007), o protective buffering tem também um impacto psicológico negativo no outro, tal como os estudos atrás referidos também indicam. Por exemplo, se o doente não partilhar as suas preocupações, o companheiro pode acabar por não corresponder às suas expectativas de apoio, não cumprindo o seu papel de cuidador, fato que frequentemente conduz a um aumento do seu próprio distress emocional. Segundo Manne et al. (2007), é por essa razão que o protective buffering quando utilizado pelo companheiro doente pode ser particularmente prejudicial para o companheiro saudável: "because it is the partner's 'job' to provide support, if the patient does not share feelings, then the partner may not know how best to provide support that matches the patient's needs and may experience concomitant feelings of inefficacy" (p.387).

Por fim, é importante considerar o impacto negativo que essa estratégia tem na própria relação. De acordo com Manne et al. (2007), quando um parceiro numa relação íntima não revela os seus sentimentos e preocupações sobre um acontecimento de vida perturbador, o processo de manutenção da intimidade é comprometido na medida em que se perde uma oportunidade para que o outro responda de forma empática e responsiva (Manne \& Badr, 2008).

\section{Conclusão}

Este estudo foi desenvolvido com o objetivo de sistematizar e integrar a informação existente na literatura sobre a comunicação, particularmente no contexto de casal, de mulheres com cancro da mama. Subjacente a esta revisão esteve a necessidade de encontrar alguma coerência nos escassos estudos desenvolvidos até o momento, bem como um quadro conceptual adequado que permitisse compreender o papel que a comunicação desempenha na adaptação ao cancro. Apesar de a comunicação ser fundamental na adaptação da doente e na manutenção de uma relação conjugal positiva, essa área tem recebido pouca atenção dos investigadores, não existindo um quadro conceptual integrador.

O presente estudo de revisão sugere que a partilha de pensamentos, preocupações e sentimentos relacionados com a doença, no contexto da relação de casal (ou outros contextos relacionais), é fundamental. Efetivamente, vários estudos mostram que a comunicação é muito importante para uma melhor adaptação da mulher à doença. Essa associação pode ser explicada por vários fatores, com destaque para o papel da comunicação como mecanismo facilitador do processamento cognitivo da experiência de cancro, tal como os modelos de processamento cognitivo dos acontecimentos traumáticos sugerem. Paralelamente, a comunicação é central para o desenvolvimento e a manutenção da intimidade entre o casal, que, por sua vez, está associada a uma maior satisfação conjugal. Contudo, e não obstante a necessidade que a maioria das doentes tem de partilhar as suas experiências, em particular com o seu companheiro, surgem, por vezes, obstáculos à comunicação. Da revisão da literatura efetuada, destacam-se os designados constrangimentos sociais e a estratégia 
de protective buffering. Esses obstáculos, ao impedirem a autorrevelação, acabam por interferir negativamente no processamento cognitivo da informação, dificultando, assim, a adaptação da doente e do seu companheiro.

Por fim, importa sublinhar a necessidade de que, futuramente, a investigação com doentes oncológicos e especificamente com cancro da mama se centre também nesta área, dada a sua relevância não só para a doente como também para o companheiro e, especialmente, para o casal como unidade interdependente. São várias as temáticas ainda não desenvolvidas. Seria importante, por exemplo, a realização de estudos longitudinais que procurassem analisar os preditores de uma comunicação positiva entre o casal ao longo do curso da doença. Tal estudo poderia identificar importantes fatores de risco e/ou de proteção, que poderiam, assim, ser considerados na prática clínica com esses casais. Simultaneamente, e não obstante alguns estudos terem já sido desenvolvidos com mulheres com cancro da mama, será com certeza importante levar a cabo um maior número de investigações com essa população específica para que o conhecimento adquirido seja mais aprofundado e específico. Os resultados de vários estudos apresentados na presente revisão, conduzidos com outros tipos de cancro, podem não ser generalizáveis a essa população específica.

Outro aspecto enfatizado no presente trabalho foram os obstáculos a uma comunicação saudável ou mesmo à sua ocorrência. Em relação aos constrangimentos sociais, a investigação existente centra-se, sobretudo, na análise dos comportamentos ou atitudes de outros elementos da rede social do doente e não tanto no(a) companheiro(a). Desse modo, a necessidade de um foco específico no casal é mais uma vez acentuada, para que seja possível compreender a relação entre os comportamentos específicos de um dos elementos da díade conjugal (afastamento, crítica etc.) e a adaptação do outro à experiência de cancro. Importante seria ainda analisar o papel mediador da inibição do processamento cognitivo nessa relação, dado que alguns estudos sugerem que os constrangimentos estão associados a diversos indicadores de inibição 104 do processamento.
Outra importante pista para a investigação futura é o estudo da relação entre a comunicação e o crescimento pós-traumático da doente. Se a partilha da experiência parece ser fundamental para o processamento e integração da experiência traumática da doença e, consequentemente, para a adaptação, importa saber se será relevante para a experiência de crescimento perante essa adversidade.

Em suma, não obstante a escassa produção científica nesta área e a sua relativa desarticulação, o presente estudo permitiu, de modo coerente e sin-tético, evidenciar o importante papel da comunicação entre o casal na adaptação a essa experiência. Ficou igualmente claro que esse processo de partilha com o outro é muito importante na integração cognitiva da experiência, pelo que deve ser fomentado. Assim, na prática clínica, parece ser importante implementar estratégias que encorajem e promovam uma partilha aberta de pensamentos, sentimentos e preocupações entre os elementos do casal. Essa partilha fomentará não só uma relação mais íntima e positiva, como também facilitará o processamento da informação relacionada com o diagnóstico de cancro, promovendo a adaptação da doente.

\section{Referências}

Clark, L. (1993). Stress and the cognitive-conversational benefits of social interaction. Journal of Social and Clinical Psychology, 12(1), 25-55.

Cordova, M., Cunningham, L., Carlson, C., \& Andrykowski, M. (2001). Social constraints, cognitive processing, and adjustment to breast cancer. Journal of Consulting and Clinical Psychology, 69(4), 706-711.

Coyne, J., Ellard, J., \& Smith, D. (1990). Social support, interdependence, and the dilemmas of helping. In B. Sarason, I. Sarason \& G. Pierce (Orgs.), Social support: An interactional view (pp.129-149). New York: Wiley.

Coyne, J., \& Smith, D. (1991). Couples coping with a myocardial infarction: A contextual perspective on wives' distress. Journal of Personality and Social Psychology, 61(3), 404-412.

Creamer, M., Burgess, P., \& Pattison, P. (1992). Reaction to trauma: A cognitive processing model. Journal of Abnormal Psychology, 101(3), 452-459.

Dunkel-Schetter, C., \&Wortman, C. (1982). The interpersonal dynamics of cancer: Problems in social relationships 
and their impact on the patient. In H. Friedman \& $\mathrm{M}$. DiMatteo (Orgs.), Interpersonal issues in health care (pp.69-100). New York: Academic Press.

Figueiredo, M., Fries, E., \& Ingram, K. (2004). The role of disclosure patterns and unsupportive social interactions in the well-being of breast cancer patients. Psycho-Oncology, 13(2), 96-105.

Foa, E., Steketee, G., \& Rothbaum, B. (1989). Behavioralcognitive conceptualizations of post-traumatic stress disorder. Behavioral Therapy, 20(2), 155-176.

Giese-Davis, J., Hermanson, K., Koopman, C., Weibel, D., \& Spiegel, D. (2000). Quality of couples' relationship and adjustment to metastatic breast cancer. Journal of Family Psychology, 14(4), 251-266.

Hagedoorn, M., Kuijer, R., Buunk, B., DeJong, M., Wobbes, T., \& Sanderman, R. (2000). Marital satisfaction in patients with cancer: Does support from intimate partners benefit those who need it the most? Health Psychology, 19(3), 274-282.

Horowitz, M. (1986). Stress response syndromes. New York: Jason Aronson.

Janoff-Bulman, R. (1992). Shattered assumptions: Towards a new psychology of trauma. New York: Free Press.

Kuijer, R., Ybema, J., Buunk, B., Jong, M., Thijs-Boer, F., \& Sanderman, R. (2000). Active engagement, protective buffering, and overprotection: Three ways of giving support by intimate partners of patients with cancer. Journal of Social and Clinical Psychology, 19(2), 256-275.

Lepore, S., \& Helgeson, V. (1998). Social constraints, intrusive thoughts, and mental health after prostate cancer. Journal of Social and Clinical Psychology, 17(1), 89-106.

Lepore, S., Ragan, J., \& Jones, S. (2000). Talking facilitates cognitive-emotional processes of adaptation to an acute stressor. Journal of Personality and Social Psychology, 78(3), 499-508.

Lepore, S., Silver, R., Wortman, C., \& Wayment, H. (1996). Social constraints, intrusive thoughts, and depressive symptoms among bereaved mothers. Journal of Personality and Social Psychology, 70(2), 271-282.

Manne, S., \& Badr, H. (2008). Intimacy and relationship processes in couples' psychosocial adaptation to cancer. Cancer, 112(11 Suppl), 2541-2555.

Manne, S., Dougherty, J., Veach, S., \& Kless, R. (1999). Hiding worries from one's spouse: Protective buffering among cancer patients and their spouses. Cancer Research Therapy and Control, 8, 175-188.
Manne, S., Norton, T., Ostroff, J., Winkel, G., Fox, K., \& Grana, G. (2007). Protective buffering and psychological distress among couples coping with breast cancer: The moderating role of relationship satisfaction. Journal of Family Psychology, 21(3), 380-388.

Manne, S., Ostroff, J., Norton, T., Fox, K., Goldstein, L., \& Grana, G. (2006). Cancer-related relationship communication in couples coping with early stage breast cancer. Psycho-Oncology, 15(3), 234-247.

Merves-Okin, L., Edmund, A., \& Frank, B. (1991). Perceptions of intimacy in marriage: A study of married couples. American Journal of Family Therapy, 19(2), 110-118.

Mesters, I., van der Born, H., McCormick, L., Pruyn, J., de Boer, M., \& Imbos, T. (1997). Openness to discuss cancer in the nuclear family: Scale, development, and validation. Psychosomatic Medicine, 59(3), 269-279.

Pennebaker, J. (1989). Confession, inhibition, and disease. In L. Berkowitz (Org.), Advances in experimental social psychology (Vol.22, pp.211-244). Orlando, FL: Academic Press.

Pistrang, N., \& Barker, C. (1992). Disclosure of concerns in breast cancer. Psycho-Oncology, 1(3), 183-192.

Pistrang, N., \& Barker, C. (1995). The partner relationship in psychological response to breast cancer. Social Science \& Medicine, 40(6), 789-797.

Porter, L., Keefe, F., Hurwitz, H., \& Faber, M. (2005). Disclosure between patients with gastrointestinal cancer and their spouses. Psycho-Oncology, 14(12), 1030-1042.

Prager, K. J. (1995). The psychology of intimacy. New York: The Guilford Press.

Rachman, S. (1980). Emotional processing. Behaviour Research and Therapy, 18(1), 51-60.

Reis, H. T., \& Patrick, B. (1996). Attachment and intimacy: Component processes. In T. Higgins \& A. Kruglanski (Orgs.), Social psychology: Handbook of basic principles. New York: The Guilford Press.

Silver, R., \& Wortman, C. (1980). Coping with undesirable events. In J. Garber \& M. Seligman (Orgs.), Human helplessness: Theory and applications. New York: Academic Press.

Spiegel, D., Bloom, J., \& Gottheil, E. (1983). Family environment as a predictor of adjustment to metastatic breast carcinoma. Journal of Psychosocial Oncology, 1(1), 33-44.

Recebido em: 6/2/2012

Aprovado em: 28/3/2012 
